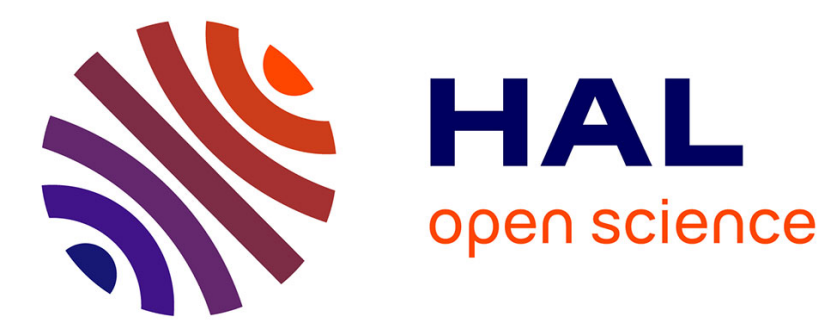

\title{
Application of a non linear local analysis method for the problem of mixed convection instability
}

Christophe Guillet, Thierry Mare, Cong Tam Nguyen

\section{To cite this version:}

Christophe Guillet, Thierry Mare, Cong Tam Nguyen. Application of a non linear local analysis method for the problem of mixed convection instability. International Journal of Non-Linear Mechanics, 2007, 42 (8), pp.981. 10.1016/j.ijnonlinmec.2007.04.004 . hal-00501755

\section{HAL Id: hal-00501755 \\ https://hal.science/hal-00501755}

Submitted on 12 Jul 2010

HAL is a multi-disciplinary open access archive for the deposit and dissemination of scientific research documents, whether they are published or not. The documents may come from teaching and research institutions in France or abroad, or from public or private research centers.
L'archive ouverte pluridisciplinaire $\mathbf{H A L}$, est destinée au dépôt et à la diffusion de documents scientifiques de niveau recherche, publiés ou non, émanant des établissements d'enseignement et de recherche français ou étrangers, des laboratoires publics ou privés. 


\section{Author's Accepted Manuscript}

Application of a non linear local analysis method for the problem of mixed convection instability

Christophe Guillet, Thierry Mare, Cong Tam Nguyen

PII:

DOI:

S0020-7462(07)00122-9

Reference:

doi:10.1016/j.ijnonlinmec.2007.04.004

NLM 1377

To appear in: International Journal of Non-

Linear Mechanics

Received date: 18 April 2007

Accepted date: 19 April 2007
INTERNATIONAL JOURNAL OF

NON-LINEAR

MECHANICS

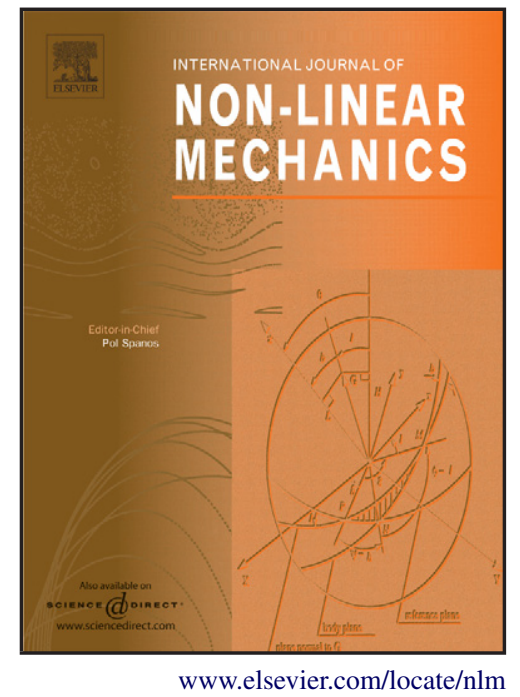

www.elsevier.com/locate/nlm

Cite this article as: Christophe Guillet, Thierry Mare and Cong Tam Nguyen, Application of a non linear local analysis method for the problem of mixed convection instability, International Journal of Non-Linear Mechanics (2007), doi:10.1016/j.ijnonlinmec.2007.04.004

This is a PDF file of an unedited manuscript that has been accepted for publication. As a service to our customers we are providing this early version of the manuscript. The manuscript will undergo copyediting, typesetting, and review of the resulting galley proof before it is published in its final citable form. Please note that during the production process errors may be discovered which could affect the content, and all legal disclaimers that apply to the journal pertain. 


\title{
Application of a non linear local analysis method for the problem of mixed convection instability
}

\author{
Christophe GUILLET ${ }^{(a)}$, Thierry $\operatorname{MARE}^{(b)}$, Cong Tam NGUYEN ${ }^{(c)}$
}

(a) Institut de Mathématiques de Bourgogne, UMR 5584 CNRS, Université de Bourgogne, 9 Avenue Alain Savary, BP 47870, 21078 Dijon Cedex, France.

(b) Laboratoire de thermique des bâtiments, GRGC INSA de Rennes 35043 Rennes, France.

(c) Faculty of engineering, Université de Moncton, Nouveau-Brunswick, E1A 3E9, Canada.

\section{E-mail address :}

christophe.guillet@u-bourgogne.fr, thierry.mare@iutsm.univ-rennes1.fr, nguyenc@umoncton.ca

Abstract: We consider the problem of laminar mixed convection flow between parallel, vertical and uniformly heated plates. Using the reduction method based on the center manifold theorem which was derived from the general theory of dynamical systems, we obtained a system of three-dimensional ordinary differential equations of amplitudes. The governing dimensionless parameters are the Prandtl, Rayleigh and Reynolds numbers. We have found that when the forcing parameter, the Rayleigh number, increases beyond the critical value $R a_{s}$, the stationary solution is a pitchfork bifurcation point of the system.

Introduction The laminar mixed convection, which is encountered in various applications in thermal engineering, received a special attention from researchers in the past decades. A large amount of research works have been produced considering various geometries. A partial review of the relevant works was presented in [1], [2], [3] for the problem of tube flow and two-dimensional channel flow. A following exhaustive review is emphasised on the flow instability.

For the tube flow in particular, one must cite the pioneering works by Hanratty and colleagues in the early sixties [4], [5] who have shown that the non-isothermal flow may become highly unstable. The flow transition from a steady laminar state to an unstable one has been observed at a relatively low value of the Reynolds number. The corresponding unstable flow structure, which was not turbulent, has shown the 'new equilibrium' state consisting of large scale, regular and periodic fluid motions.

These authors have also found that for heated upward flow (assisted-buoyancy), instabilities occur when the velocity profile develop points of inflexion. For the heated downward flow (opposed buoyancy case), the instability seems to be caused by the boundary layer detachment. Wang and colleagues [6] have investigated the problem of mixed convection with flow reversal in the thermal entrance region of horizontal and vertical pipes at low Péclet number. For a vertical tube flow, they have found that the flow reversal occurs at the pipe center for the heating case and near the tube wall for the cooling case. Such reversal occurs at relatively high $-\mathrm{Gr} / \mathrm{Re}$ - ratio. Different regimes of reversed flow have 
been identified in the Pe- $-\mathrm{Gr} / \mathrm{Re}$ - coordinate map. The phenomenon of bifurcation in a horizontal tube has also been investigated [7] the existence of two-cell and four-cell solutions has been demonstrated.

With regard to the two-dimensional laminar mixed convection flow between parallel planes, Chang and Lin [3] and Lin et al. [8] have shown that the flow may become unstable i.e. oscillatory, which possesses a single fundamental frequency when the ratio $G r / R e^{2}$ exceeds a critical value. With further increasing of the opposing buoyancy, a second fundamental frequency is observed and the flow becomes quasi-periodic. Their numerical results have also shown that increasing the Reynolds number tends to stabilize the flow. The thermal instability has also been investigated for flow in a horizontal channel $([9])$.

The first theoretical study of the flow stability in mixed laminar convection was due to Nandakumar [10] who has established the existence of dual solutions, the two-vortex and the four-vortex flow patterns. The bifurcation phenomenon is seen as a two-parameter problem for a rectangular duct. For a circular cross-section duct, it consists of a oneparameter problem that exhibits similar features to those observed for the classical Dean problem.

On the other hand, Yao [11], [12] has performed a linear stability analysis for a flow of water in a heated vertical tube. He has found that the fully developed non-isothermal flow appears highly unstable, and the 'bifurcated equilibrium state' is likely to be a doublehelices structure. Such unstable structure seems to be susceptible to delay the flow transition to turbulence. In a recent linear stability analysis, Su and Chung [13] have shown that the mixed laminar convection flow in a vertical tube may become unstable at relatively low Reynolds number and Rayleigh numbers, and this independently of the Prandtl number. For water in particular, the predicted values for the critical Rayleigh number were comparable to the corresponding experimental data by Scheele and Hanratty [5] for both assisted and opposed buoyancy cases. Su and Chung [13] have also found that the Prandtl number has important effect on the stability in the case of assisted buoyancy flow as it can change the flow instability mechanism. For Prandtl number less than 0.3, the thermal-shear instability is dominant while for $\operatorname{Pr}>0,3$, the assisted-thermal-buoyant instability becomes preponderant. Similar theoretical studies of the flow stability were also performed for the case of two-dimensional channel. Paolucci et al. [14], using an integral Chebyshev pseudo-spectral method, have found that the two dimensional disturbances are the most unstable. Recently, Chen and Chung [15], [16] studied the stability of a differentially heated vertical channel for various Prandtl numbers. Their results have shown that both the Prandtl number and Reynolds number hold very important effects on the critical Grashof number, the wave number, the wave speed as well and the instability mechanism for high Prandtl number fluids. The existence of multiple local minimum wave numbers has been determined, which are believed to be responsible of the sudden jumps observed on the critical wave number and wave speed. It is important to mention that most of the above theoretical studies were based on the classical linear stability analysis.

In the present work, we have revisited the two-dimensional problem of the flow stability and bifurcation for a fully developed laminar mixed convection flow. For doing this, we have employed the non-linear analysis method that was derived from the general theory of dynamical systems .

We have considered the ascendant flow of a viscous fluid between parallel, vertical and uniformly heated planes. At the laminar state, a constant heat flux condition is imposed at both planes. The problem is described by three dimensionless parameters: the Prandtl 
(Pr) number characterizing the fluid, the Reynolds (Re) number representing forced convection and the Rayleigh ( $\mathrm{Ra}$ ) number characterizing the buoyancy forces due to the heating effect. The Rayleigh number is the only parameter that is varying in this study. The objective of our study is to show, using such a simple fluid flow problem, that we can introduce some tools obtained from the dynamical system theory - the center manifold theorem - to qualitatively describe the system in the vicinity of a bifurcation. In fact, as shown in the presented results, we have proved that there is a pitchfork bifurcation in the system for a critical value of Ra, which is independent of $\operatorname{Pr}$ and Re and some infinitesimal perturbation of the stationary state.

\section{The center manifold theorem}

The center manifold theorem is a tool of the dynamical systems theory used in the non linear evolution problems where partial differential equations and infinite degrees of freeedom are involved, such as in the domain of fluid mechanics. Thus, by choosing a appropriate functional scheme that transforms a system of Navier-Stokes equations into a set of ordinary differential equations such as

$$
\frac{d X}{d t}=F(\mu, X)
$$

where $X$ is a vector describing the state variables and $\mu$ is a bifurcation parameter, the center manifold theorem allows us not only to reduce the dimension of such a system in the vicinity of a bifurcation, but also to be able to analyse the type and the local dynamics of such bifurcation. It is important to note that although the solutions of a non linear ODE system in the neighbourhood of a bifurcation point can be expressed in a basis of eigenvectors for the linearized system, their asymptotic behaviour is only given by a finite number of these vectors. The center manifold theorem stipulates that if a linearized system in the neighbourhood of a bifurcation point has a discrete spectrum with $\mathrm{n}$ eigenvalues on the imaginary axis and all the remaining part lies within the left-hand side of the complex plane, then the asymptotic behaviour of the solutions close to this critical point is given by $n$ ordinary differential equations. Moreover, the form of these equations depends only on the type of the singularity.

\section{Mathematical modelling}

In this study, we assume that the fluid is incompressible with constant physical properties i.e. fluid density, thermal conductivity, thermal diffusivity and viscosity are temperature and pressue dependent, with an exception of the fluid density that appears in the buoyancy forces. This density is assumed to vary linearly with temperature according to the Oberbeck-Boussinesq approximation, which is often referred by many authors dealing with the buoyancy-driven flows as the Boussinesq approximation. In fact, before Boussinesq's work in 1903, Oberbeck had come up with the same approximation (see [17]). It is interesting to mention that, by using a perturbation technique to studying the thermal response of a linearly viscous, mechanically incompressible but thermally compressible fluid, Rajagopal et al. [17] have eloquently derived the Oberbeck-Boussinesq approximation as a third-order perturbation. It is worth to mention from their works that one can also obtain 
'higher order' approximations to the equations. Under these assumptions, the equations of continuity, momentum and energy of the physical system are given as follows:

$$
\begin{array}{r}
\nabla \cdot \vec{V}=0 \\
\rho_{0}\left(\frac{\partial \vec{V}}{\partial t}+(\vec{V} \cdot \nabla) \vec{V}\right)=-\nabla p+\mu \nabla^{2} \vec{V}+\rho \vec{g} \\
\frac{\partial T}{\partial t}+(\vec{V} \cdot \nabla) T=\chi \nabla^{2} T
\end{array}
$$

where the gravitational acceleration $\vec{g}$ and the velocity field $\vec{V}$ are opposed, $p$ is the fluid pressure, $\mu$ the fluid dynamic viscosisty, $\chi$ the thermal diffusivity and $\rho$ the fluid density which is given by

$$
\rho=\rho_{0}\left[1-\beta\left(T-T_{0}\right)\right]
$$

where $T$ is the local fluid temperature.

We assume that the fluid flow between two parallel vertical plates separated by a distance $D$ is described by a two-dimensional velocity field $\vec{V}=\left(V_{x}, V_{z}\right)$, see Figure 1. Moreover, the wall temperature is given by $T_{W}(z)=T_{W_{0}}+C_{1} D z$ where $T_{W_{0}}$ is the reference wall temperature assumed to be equal to $T_{0}$ and $C_{1}$ is a constant which is the axial gradient of wall temperature; we have considered that $C_{1}$ is positive i.e. the flow is buoyancyassisted.

The number of parameters can be reduced by introducing the following dimensionless variables as follows:

$$
\begin{array}{cc}
x^{*}=\frac{x}{D} & z^{*}=\frac{z}{D} \\
V_{x}^{*}=\frac{V_{x}}{V_{0}} & V_{z}^{*}=\frac{V_{z}}{V_{0}} \\
p^{*}=\frac{p}{\rho_{0} V_{0}^{2}} & T^{*}=\frac{\left(T-T_{W_{0}}\right)+C_{1} D z^{*}}{\operatorname{RePr} C_{1} D} \\
t^{*}=\frac{V_{0} t}{D} &
\end{array}
$$

where $V_{0}$ is the mean velocity, the Prandtl and Reynold numbers Pr and Re are given by

$$
\begin{array}{r}
\operatorname{Pr}=\frac{\mu}{\rho_{0} \chi} \\
R e=\frac{\rho_{0} V_{0} D}{\mu}
\end{array}
$$

The following dimensionless governing equations are then obtained:

$$
\begin{array}{r}
\nabla \cdot \vec{V}^{*}=0 \\
\frac{\partial \vec{V}^{*}}{\partial t^{*}}+\left(\vec{V}^{*} \cdot \nabla\right) \vec{V}^{*}=-\nabla p^{*}+\frac{1}{R e} \nabla^{2} \vec{V}^{*}+\gamma\left(T^{*}, z^{*}\right) \vec{g} \\
\frac{\partial T^{*}}{\partial t^{*}}+\left(\vec{V}^{*} \cdot \nabla\right) T^{*}=\frac{1}{\operatorname{RePr}} \nabla^{2} T^{*}+\frac{1}{\operatorname{RePr}} V_{z}^{*}
\end{array}
$$


where

$$
\gamma\left(T^{*}, z^{*}\right)=\frac{D}{V_{0}^{2}}\left(1-\beta C_{1} D\left(z^{*}-\operatorname{Re} \operatorname{Pr} T^{*}\right)\right)
$$

and $\nabla^{2}=\frac{\partial^{2}}{\partial x^{* 2}}+\frac{\partial^{2}}{\partial z^{* 2}}$ is is the Laplacian operator in the Cartesian coordinate system. The boundary conditions are identical to those considered by Chen et Chung [16]:

$$
T^{*}=V^{*}=0 \quad\left(x^{*}= \pm \frac{1}{2}\right)
$$

\section{Study of a simplified model}

\subsection{Reduction of the system of Navier-Stokes equations}

From the mathematical point of view, the difficulty resides in finding a suitable functional framework that takes into consideration of the continuity equation and the pressure term in the equations of momentum in order to reduce the Navier-Stokes system. For the present case, we assumed that there is a dimensionless stream function $\Psi^{*}$ given by:

$$
\vec{V}^{*}=\left(V_{x^{*}}, V_{z^{*}}\right)=\left(\frac{\partial \Psi^{*}}{\partial z^{*}},-\frac{\partial \Psi^{*}}{\partial x^{*}}\right)
$$

such that the continuity equation is satisfied.

By eliminating the pressure term by cross differentiating the equation of conservation of momentum (13), and by using (15)-(17), the dimensionless equations of motion then become

$$
\begin{array}{llll}
\frac{\partial}{\partial t^{*}}\left(\nabla^{2} \Psi^{*}\right) & = & \frac{1}{R e} \nabla^{4} \Psi^{*}-\frac{R a}{R e} \frac{\partial T^{*}}{\partial x^{*}} & -J\left(\Psi^{*}, \nabla^{2} \Psi^{*}\right) \\
\frac{\partial T^{*}}{\partial t^{*}} & =\frac{1}{\operatorname{RePr}} \nabla^{2} T^{*}-\frac{1}{\operatorname{RePr}} \frac{\partial \Psi^{*}}{\partial x^{*}} & -J\left(\Psi^{*}, T^{*}\right)
\end{array}
$$

where the operator $J$ is defined as $J(f, g)=\frac{\partial f}{\partial z} \frac{\partial g}{\partial x}-\frac{\partial f}{\partial x} \frac{\partial g}{\partial z}$.

The new dimensionless parameter appearing in these equations is the Rayleigh number given by

$$
R a=\frac{g \beta C_{1} D^{4}}{\chi \nu}
$$

where $\nu=\frac{\mu}{\rho_{0}}$.

In order to apply our method of reduction, we have considered the following boundary conditions

$$
\frac{\partial T^{*}}{\partial x^{*}}=\frac{\partial \psi^{*}}{\partial z^{*}}=0 \quad\left(x^{*}= \pm \frac{1}{2}\right)
$$

Under the above conditions, it can be possible to find a stationary state $S_{B}=\left(\psi_{B}, T_{B}\right)$ that is independent of $\mathrm{z}$ and satisfies the following ordinary differential equations

$$
\begin{aligned}
\frac{d^{(5)} T_{B}}{d t^{5}} & =R a \frac{d T_{B}}{d x} \\
\psi_{B} & =\frac{d T_{B}}{d x}
\end{aligned}
$$

The trivial function is of course the solution of (21) for all Ra, which is also the solution chosen to describe our method. The aim of the study consists of describing qualitatively 
the temporal evolution of an infinitesimal perturbation $S^{\prime}=\left(\psi^{\prime}, T^{\prime}\right)$ for the zero stationary solution $S_{B}=(0,0)$ under the hypothesis that the Rayleigh number is the only forcing parameter.

\subsection{Amplitude equations}

We choose the following minimal representation for which the temporal and spatial parts are uncoupled so that the spectral conditions for the center manifold theorem are satisfied

$$
\begin{array}{r}
\Psi^{\prime}(x, z, t)=A(t) \sin (\pi \alpha x) \sin (\pi z) \\
T^{\prime}(x, z, t)=B(t) \cos (\pi \alpha x) \sin (\pi z)+C(t) \sin (2 \pi z)
\end{array}
$$

Such equations satisfy the imposed boundary conditions (20) for $\alpha=2$. Thus, by substituting the expressions (22)-(23) into the system (18), we obtain the following non linear system of amplitude equations:

$$
\frac{d X}{d t}=F(\mu, X)
$$

where $X=(A, B, C)$ and $\mu=R a$ with $F(\mu, X)=L_{\mu} X+N(X)$ where the linear operator $L_{\mu}$ is defined by

$$
L_{\mu}=\left(\begin{array}{lll}
-\frac{K^{2}}{R e} & -\frac{\pi \alpha R a}{K^{2} R e} & 0 \\
-\frac{\pi \alpha}{\operatorname{RePr} r} & -\frac{K^{2}}{\operatorname{RePr}} & 0 \\
0 & 0 & -\frac{4 \pi^{2}}{\operatorname{RePr}}
\end{array}\right)
$$

and $N$ is the quadratic operator defined by

$$
N(X)=\left(\begin{array}{l}
0 \\
-2 \pi^{2} A C \\
\frac{\pi^{2} \alpha}{2} A B
\end{array}\right)
$$

\subsection{Linearization of the system}

At first, the linear analysis allows us to prove the existence of fixed points that will be considered for bifurcation points. Such linear analysis cannot, unfortunately, provides local information about the solutions that appear near the critical point. Therefore, the non linear analysis will be necessary as it is capable to overcome such problem.

Thus, the fixed points or the steady states $X=(A, B, C)$ of the system (24) are given by

$$
\text { A } \begin{array}{rlc}
\left(\pi^{4} \alpha^{3} A^{2}-\frac{K^{2} \alpha}{R e} \operatorname{det}\left(L_{\mu}\right)\right. & =0 \\
B & = & -\frac{K^{4}}{\pi \alpha R a} A \\
C & = & \frac{\alpha R e P r}{8} A B
\end{array}
$$

where

$$
\operatorname{det} L_{\mu}=\frac{4 \pi^{4} \alpha^{2}}{K^{2} R e^{3} \operatorname{Pr}^{2}}\left(R a-\frac{K^{6}}{\pi^{2} \alpha^{2}}\right)
$$


is the determinant of the matrix $L_{\mu}$.

We can deduce that $X=(0,0,0)$ is a fixed point - or a steady state - of the system $(24)$ for all choice of $\mu$. By solving (27), one can see that the systems possesses two other symmetric fixed points if and only if

$$
R a>\frac{K^{6}}{\pi^{2} \alpha^{2}}
$$

In a first step, we are going to investigate the linearized problem around $X=(0,0,0)$ for which we have:

$$
\frac{d X}{d t^{*}}=L_{\mu} X
$$

The spectrum of the linear operator $L_{\mu}$ is the roots of the polynomial $P_{L}$ defined by

$$
P_{L}(\lambda)=\left|\begin{array}{lll}
-\frac{K^{2}}{R e}-\lambda & -\frac{\pi \alpha R a}{K^{2} R e} & 0 \\
-\frac{\pi \alpha}{\operatorname{RePr}} & -\frac{K^{2}}{\operatorname{RePr}}-\lambda & 0 \\
0 & 0 & -\frac{4 \pi^{2}}{\operatorname{RePr}}-\lambda
\end{array}\right|
$$

which can be written under a factorized form as follows:

$$
P_{L}(\lambda)=\left(-\frac{4 \pi^{2}}{\operatorname{RePr}}-\lambda\right)\left[\lambda^{2}+(1+\operatorname{Pr}) \frac{K^{2}}{\operatorname{RePr}} \lambda+\left(\frac{K^{4}}{\operatorname{Pr} R e^{2}}-\frac{\pi^{2} \alpha^{2} R a}{K^{2} \operatorname{Pr} R e^{2}}\right)\right]
$$

Such result proves that $X=(0,0,0)$ is a candidate to a primary steady state bifurcation if and only if 0 is a simple eigenvalue of $L_{\mu}$. This is possible when the condition

$$
\frac{K^{4}}{\operatorname{Pr} R e^{2}}-\frac{\pi^{2} \alpha^{2} R a}{K^{2} \operatorname{Pr} R e^{2}}=0
$$

is satisfied, which results to $R a=R a_{s}$ where the critical Rayleigh number $R a_{s}$ is given as:

$$
R a_{s}=\frac{K^{6}}{\pi^{2} \alpha^{2}}
$$

so approximately $R a_{s} \approx 3043,68$. We will study the system in the neighbourhood of this fixed point. It is interesting to note that the existence of the other type of primary bifurcation - the Hopf bifurcation- can be proven to be impossible as the condition

$$
(1+\operatorname{Pr}) \frac{K^{2}}{\operatorname{RePr}}=0
$$

will never be satisfied. 


\subsection{Reduction of the system on a center manifold}

In the following, we consider the part of the matrix $L_{\mu}$ which is dependent on the parameter $\mu$ as a non linear term.

By introducing $\epsilon=R a-R a_{s}$, we can write

$$
L_{\mu}=L\left(R a_{s}\right)+\tilde{L}(\epsilon)
$$

where

$$
\tilde{L}(\epsilon)=\left(\begin{array}{lll}
0 & -\frac{\pi \alpha \epsilon}{K^{2} R e} & 0 \\
0 & 0 & 0 \\
0 & 0 & 0
\end{array}\right)
$$

The system (24) can then be rewritten as:

$$
\frac{d X}{d t^{*}}=L\left(R a_{s}\right) X+\tilde{g}(X, \epsilon)
$$

where

$$
\tilde{g}(X, \epsilon)=\tilde{L}(\epsilon) X+\left(\begin{array}{l}
0 \\
-2 \pi^{2} \alpha A C \\
\frac{\pi^{2} \alpha}{2} A B
\end{array}\right)
$$

Once the condition (33) is satisfied, the eigenvalues of $L\left(R a_{s}\right)$ are

$$
\lambda_{1}=0, \quad \lambda_{2}=-(1+\operatorname{Pr}) \frac{K^{2}}{\operatorname{RePr}}, \quad \lambda_{3}=\frac{-4 \pi^{2}}{\operatorname{RePr}}
$$

Then we associate an eigenvector $V_{i}$ to the eigenvalue $\lambda_{i}$ for $i=1,2,3$ such that the system (38) can be written as

$$
\frac{d Y}{d t^{*}}=M\left(R a_{s}\right) Y+\widetilde{h}(Y, \epsilon)
$$

where $M\left(R a_{s}\right)=P^{-1} L\left(R a_{s}\right) P$ is the diagonal matrix defined by

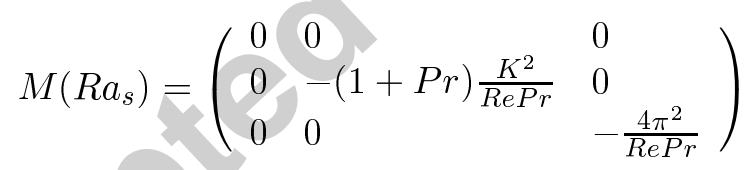

with

$$
P=\left(\begin{array}{lll}
-\frac{K^{2}}{\pi \alpha} & \operatorname{Pr} \frac{K^{2}}{\pi \alpha} & 0 \\
1 & 1 & 0 \\
0 & 0 & 1
\end{array}\right)
$$

which is an invertible matrix defining the linear variation of coordinates

$$
Y=P^{-1} X
$$

and

$$
\tilde{h}(Y, \epsilon)=P^{-1} \tilde{g}(P Y, \epsilon)
$$


is defined by

$$
\tilde{h}(Y, \epsilon)=\tilde{M}(\epsilon) Y+\left(\begin{array}{l}
2 \frac{\pi P r K^{2}}{(1+P r)}(U W-\operatorname{Pr} V W) \\
2 \frac{\left.\pi K^{2}\right)}{(1+P r)}(U W-\operatorname{Pr} V W) \\
-\frac{\pi K^{2}}{2}\left(U^{2}+(1-\operatorname{Pr}) U V-\operatorname{Pr} V^{2}\right)
\end{array}\right)
$$

where

$$
\tilde{M}(\epsilon)=\frac{\pi^{2} \alpha^{2} \epsilon}{(1+\operatorname{Pr}) \operatorname{Re}}\left(\begin{array}{lll}
1 & 1 & 0 \\
0 & -1 & 0 \\
0 & 0 & 0
\end{array}\right)
$$

If we consider $Y=(U, Z)$ with $Z=(V, W)$, then the system (41) becomes

$$
\begin{array}{lcc}
\frac{d U}{d t^{*}}= & \tilde{r}(U, Z, \epsilon) \\
\frac{d Z}{d t^{*}}= & B Z+\tilde{s}(U, Z, \epsilon)
\end{array}
$$

with

$$
B=\left(\begin{array}{ll}
-(1+\operatorname{Pr}) \frac{K^{2}}{\operatorname{RePr}} & 0 \\
0 & -\frac{4 \pi^{2}}{\operatorname{RePr}}
\end{array}\right)
$$

and

$$
\begin{aligned}
& \tilde{r}(U, Z, \epsilon)=\frac{\pi^{2} \alpha^{2} \epsilon}{\operatorname{Re}(1+\operatorname{Pr})}(U+V) \quad+\frac{2 \pi \operatorname{Pr} K^{2}}{(1+\operatorname{Pr})}(U W-\operatorname{Pr} V W)
\end{aligned}
$$

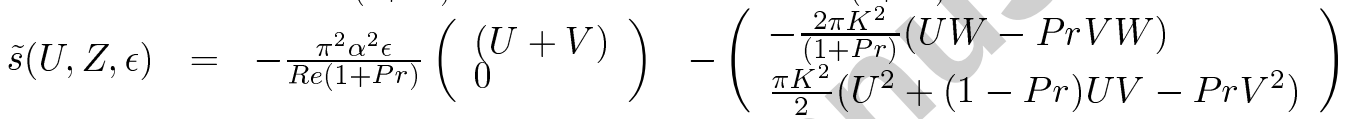

In the new coordinate system $(U, V, W)$, the directions given by the eigenvectors $V_{2}$ and $V_{3}$ are respectively defined by $V=0$ and $W=0$. For any fixed $\epsilon$ close to 0 , the central direction is given by $V_{1}$ and defined by $U=0$. By [18], we can find a one-dimensional invariant manifold for the system (41) passing by $Y=(0,0,0)$, which is tangent to the center eigenspace generated by $V_{1}$. We denote by $W_{\epsilon}^{c}(0)$ the curve called center manifold. As the center eigenspace defined by the equation $Z=0, W_{\epsilon}^{c}(0)$ is locally represented by a graph:

$$
W_{\epsilon}^{c}(0)=\left\{(U, Z) \in R^{3}\left|Z=h_{\epsilon}(U),\right| U \mid<\delta_{1}, h_{\epsilon}(0)=0, D h_{\epsilon}(0)=0\right\}
$$

such that the asymptotic dynamical behavior of the system (41) on $W_{\epsilon}^{c}(0)$ is given by the ordinary differential equation

$$
\frac{d U}{d t^{*}}=\tilde{r}\left(U, h_{\epsilon}(U), \epsilon\right)
$$

for $U$ close to 0 . To find the center manifold, we need to solve the following quasilinear partial differential equation

$$
\mathcal{N}\left(h_{\epsilon}(U)\right)=D h_{U}\left(\tilde{r}\left(U, h_{\epsilon}(U), \epsilon\right)-B h_{\epsilon}(U)-\tilde{s}\left(U, h_{\epsilon}(U), \epsilon\right)=0\right.
$$


As it is difficult to solve (53), we compute an approximate solution in assuming that $V=h_{\epsilon, 1}(U)$ and $W=h_{\epsilon, 2}(U)$ are defined by

$$
\begin{aligned}
& h_{\epsilon, 1}(U)=a_{1} U^{2} \quad+a_{2} U \epsilon+a_{3} \epsilon^{2}+\mathcal{O}\left(\|(U, \epsilon)\|^{3}\right) \\
& h_{\epsilon, 2}(U)=b_{1} U^{2}+b_{2} U \epsilon+b_{3} \epsilon^{2}+\mathcal{O}\left(\|(U, \epsilon)\|^{3}\right)
\end{aligned}
$$

Then, by substituting (54) into (53), and equating terms of identical powers to zero, we obtain:

$$
\begin{aligned}
& U^{2} \quad:(1+P r) \frac{K^{2}}{R e P r} a_{1}=0 \quad \Rightarrow a_{1}=0 \\
& \frac{4 \pi^{2}}{\operatorname{RePr}} b_{1}+\frac{\pi K^{2}}{2}=0 \quad \Rightarrow b_{1}=-\frac{K^{2} R e P r}{8 \pi}
\end{aligned}
$$

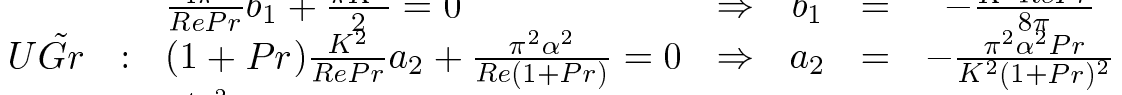

$$
\begin{aligned}
& \begin{array}{llll}
\frac{4 \pi^{2}}{\operatorname{RePr}} b_{2}=0 & \Rightarrow b_{2} & = & 0
\end{array}
\end{aligned}
$$

Using (55), we deduce that:

$$
\begin{aligned}
& h_{\epsilon, 1}(U)=-\frac{\pi^{2} \alpha^{2} P r}{K^{2}(1+P r)^{2}} U \epsilon+\mathcal{O}\left(\epsilon^{2},|U| \epsilon^{2},|U|^{3}\right) \\
& h_{\epsilon, 2}(U)=-\frac{K^{2} \operatorname{RePr}}{8 \pi} U^{2}+\mathcal{O}\left(\epsilon^{2},|U| \epsilon^{2},|U|^{3}\right)
\end{aligned}
$$

Substituting (56) in (52), we obtain the vector field reduced on $W_{\epsilon}^{c}(0)$ :

$$
\frac{d U}{d t^{*}}=\frac{U}{R e(1+P r)}\left(\pi^{2} \alpha^{2} \epsilon-\frac{K^{4} R e P r^{2}}{4} U^{2}\right)+\mathcal{O}\left(\epsilon^{2},|U| \epsilon^{2},|U|^{3}\right)
$$

\subsection{Dynamics of the solutions on the center manifold}

By neglecting higher order terms such as $\mathcal{O}\left(\epsilon^{2}\right), \mathcal{O}\left(|U| \epsilon^{2}\right), \mathcal{O}\left(|U|^{3}\right)$, etc..., we can show that the system $(57)$ has two curves of fixed points passing through $\left(R a_{s}, 0\right)$ for which the equations are respectively, $U=0$ and $U= \pm g(\mu)$ where $g$ is defined by

$$
g(\mu)=\frac{2 \pi \alpha}{K^{2} \operatorname{RePr}} \sqrt{\epsilon}
$$

For $\epsilon<0$ i.e. $R a<R a_{s}$, there is only one fixed point on the curve $U=0$ that is stable. The instability occurs when $\epsilon>0$ or $R a>R a_{s}$. At the particular point of exchange of stability $\left(R a_{s}, 0\right)$, two new stable fixed points are created and are given by $U= \pm g(R a)$. Thus, we deduce that the fixed point $\left(R a_{s}, 0\right)$ is clearly a point of pitchfork bifurcation. So we easily obtain the bifurcation diagram on the center manifold in the $R a-U$ plane as shown in Figure 2 . Thus, for $R a<R a_{s}$, the steady state $\left(\Psi^{*}, T^{*}\right)=(0,0)$ is asymptotically stable, and when $R a$ exceeds $R a_{s}$, this steady state becomes unstable. Moreover, the existence of a pitchfork bifurcation point implies that, for $R a$ close to $R a_{s}$ such as $R a>R a_{s}$, all the solutions asymptotically end up to one of the symmetric stable steady states $\left(\Psi^{*}, T^{*}\right)$ which are close to $(0,0)$. 


\section{Discussion and conclusion}

This result valid for $R e \neq 0$ has been found to be in good agreement with the numerical results by Chen and Chung [15], [16] who, by performing the linear stability analysis for similar problem, have observed the existence of such bifurcations for certain values of the parameters Re and Ra. In particular, they proved, by plotting the neutral stability curves on the $(R e, R a)$ plane, that the critical Rayleigh number $R a_{s}$ is nearly constant - which is approximately 15 - for $R e>200$ and $\operatorname{Pr}$ ranging from 0.7 to 100 . On the other hand, for small values of Re, they found that this critical value can increase to about 200 . From our non linear approach presented in this paper, we have also found that the critical Rayleygh number $R a_{s}$ is independent of $\operatorname{Pr}$ and $R e$. Furthermore, from the neutral stability curve given by $R a=R a_{s}$ in Figure 3, we have estimated $R a_{s} \simeq 3043,68$ that represents the instability boundary on the $(R e, R a)$ plane for this range of Prandtl numbers. This critical value $R a_{s} \approx 3043,68$ corresponds in fact to the value of 190,7 according to the notations of Chen and Chung. It is worth to mention that, because of simplifications of the boundary conditions used, a more complete comparison with other results was not possible at this stage.

We though that the above reduction method based on the center manifold, which has successly been applied to a simplified case, can be extended to a more comlex problem governed by three-dimensional Navier-Stokes equations (3). In order to do this, some difficulties must be solved. Thus, we must first find a suitable functional framework in order to reduce the the system of Navier-Stokes equations to a system of ordinary differential equations (see [19]). Once this first step is done, it is necessary to identify the spectrum of the associated linear operator $L_{\mu}$ in such a way that the hypotheses of the center manifold theorem are satisfied. Such a challenging and interesting task may be accomplished by using an appropriate numerical method, which are under consideration by our research group and will constitute the subject of a future paper.

Finally, it is worth to mention that our non linear approach for treating the problem of instability in mixed convection laminar flows can be, of course, extended to other geometrical configurations. Thus, in [20], an interesting result obtained for a two-dimensional axisymmetric tube flow seems to corroborate very well with the numerical results by Nguyen et al. [21] who have studied a similar mixed convection problem inside a cylindrical liquidbridge. Their 2D-axis-symmetrical model did not show any sign of the bifurcation and instabilities even at every high value of the forcing parameter that is the thermocapillary Reynolds number. On the other hand,for a full three-dimensional laminar mixed flow problem inside a vertical liquid-bridge for which many numerical results, see for example Bazzi et al. [22], have clearly shown the existence of the bifurcation phenomenon. Such a full three-dimensional model will be considered in the next steps of our research program.

Acknowledgements The authors are grateful to Prof. Mariana HARAGUS , member of the "Partial differential equations" group in the Mathematics Laboratory of the Université de Franche Comté (Besancon,France) and to Prof. Nicolas GALANIS, member of the Mechanical Department at the Université de Sherbrooke (Quebec,Canada) for their valuable comments about this work. 


\section{References}

[1] Sundén, B., Comini, G., Computational Analysis of Convection Heat Transfer, Chap. 8, WIT Press, Boston, USA (2000)

[2] Jackson, J. D., Cotton, M. A., Axcell, B. P., Study of mixed convection in vertical tubes, Int. J. Heat and Fluid Flow, Vol. 10 (1), 2-15 (1989)

[3] Chang, T.-S., Lin, T.-F., Steady and oscillatory opposing mixed convection in a symmetrically heated vertical channel with a low-Prandtl number fluid, Int. J. Heat Mass Transfer, Vol. 36, No. 15, 3783-3795 (1993)

[4] Hanratty, T. J., Rosen, E. M., Kabel, R. L.,Effects of heat transfer on flow field at low Reynolds numbers in vertical tubes, Indust. Engng. Chem., Vol. 50 (5), 815-820 (1958)

[5] Scheele, G. F., Hanratty, T. J., Effect of natural convection on stability of flow in vertical pipe, J. Fluid Mech., Vol. 14, 244-256 (1962)

[6] Wang, M., Tsuji, T., Nagano, Y.,Mixed convection with flow reversal in the thermal entrance region of horizontal and vertical pipes, Int. J. Heat Mass Transfer, Vol. 37, No. 15, 2305-2319 (1994)

[7] Orfi, J., Galanis, N., Nguyen, C.T.,Bifurcation in steady laminar mixed convection flow in uniformly heated inclined tubes, Int. J. Num. Met. Heat Fluid Flow, Vol. 9, No. 5, 543-567 (1999)

[8] Lin, T.-F, Chang, T.S., Chen, Y.-F.Development of oscillatory asymmetric recirculating flow in transient laminar opposing mixed convection in a symmetrically heated vertical channel, J. Heat Transfer, Vol. 115, No. 2, 342-352 (1993)

[9] Lee, F.S., Hwang, G.J., Transient analysis on the onset of thermal instability in the thermal entrance region of a horizontal parallel plate channel, J. Heat Transfer, Vol. 113, No. 2, 363-370 (1991)

[10] Nandakumar, K., Masliyah, J.H., Law, H.-S., Bifurcation in steady laminar mixed convection flow in horizontal ducts, J. Fluid Mech., Vol. 152, 145-161 (1985)

[11] Yao, L. S., Is a fully-developed and non-isothermal flow possible in a vertical pipe?, Int. J. Heat Mass Transfer, Vol. 30, No. 4, 707-716 (1987a)

[12] Yao, L. S.,Linear stability analysis of opposed convection in a vertical pipe, Int. J. Heat Mass Transfer, Vol. 30, 810-811 (1987b)

[13] Su, Y.-C., Chung, J. N., Linear stability analysis of mixed convection flow in a vertical pipe, J. Fluid Mech., Vol. 422, 141-166 (2000)

[14] Paolucci, S., Suslov, S.A., Vasilyev, O.V.,Stability of mixed convection flow in a differentially heated vertical channel with large temperature difference, ASME Heat Transfer Div Publ HTD Vol. 274, 33-40 (1994) 
[15] Chen, Y.-C., Chung, J.N., Stability of mixed convection in a differentially heated vertical channel, J. Heat Transfer, Vol. 120, No. 1, 127-132 (1998)

[16] Chen, Y.-C., Chung, J.N.,Linear stability of mixed convection in a vertical channel flow, J. Fluid Mech., Vol. 325, 29-51 (1996)

[17] Rajagopal, K. R., Ruzicka, M., Srinivasa, A.R., On the Oberbeck-Boussinesq approximation, Mathematical Models and Methods in Applied Sciences, Vol. 6, No. 8, 1157-1167 (1996).

[18] Wiggins S.,Introduction to Applied Nonliear Dynamical Systems and Chaos, Springer Verlag (1990)

[19] Chossat P., Iooss G., The Couette-Taylor Problem, Applied mathematical sciences; Vol. 102, Springer Verlag (1994)

[20] Guillet C.Instabilité de systèmes Hamiltoniens au sens de Chirikov et bifurcation dans un problème d'évolution non linéaire issu de la Physique, Thesis, Université de Franche Comté, (2004)

[21] Nguyen, C.T., Orfi, J., Bazzi, Transient behavior of a $\mathrm{NaNO}_{3}$ float zone operating at high Marangoni number under $\mu-g$ conditions, Numerical Heat Transfert, Part A, Vol. 28, pp. 299-320 (1995)

[22] Bazzi, H., Galanis, N., Nguyen, C.T.,Numerical study of the unstable thermocapillary flow in a sislicon float zone under $m u-g$ condition, Int. J. Therm. Sci., Vol.40, 702-716 (2001)

[23] Arnold V.I.Loss of stability of self-oscillations close to resonance and versal deformations of equivariant vector fields,Functional Anal. and Applics, 11, 85-92 (1977)

[24] Dautray R., Lion J-L.,Analyse mathématique et calcul numérique pour les sciences et techniques, Vol.8, Masson (1988)

[25] Golubitsky M., Stewart I., The Symmetry Perspective,Birkhäuser Verlag (2002) Int. J. Heat and Fluid Flow, Vol. 10 (1), 2-15 (1989)

[26] Guckenheimer J., Holmes P.J.,Nonlinear Oscillations, Dynamical Systems, and Bifurcations of Vector Fields, Springer Verlag (1983)

[27] Guckenheimer J., Knobloch E.,Nonlinear Convection in a Rotating Layer: Amplitude Expansions and Normal Forms, Geophys, Astrophys.Fluid Dynamics, Vol.23, 247-272 (1983)

[28] Siddheshwar P.G., Pranesh S.,An analytical study of linear and non-linear convection in Boussinesq-Stokes suspensions, International Journal of Non Linear Mechanics (2002)

[29] Subramanian S.,Balakotaiah V.,Mode Interactions in Reaction-Driven Convection in a Porous Medium, Chemical Engineering Science, Vol.50, n12,1851-1866 (1995) 


\section{List of Figures}

$1 \quad$ Schematic representation of system under study :Two parallel vertical plates 15

2 Bifurcation diagram on the center manifold in the $R a-U$ plane . . . . . . 16

3 The instability boundary on the Re - Ra plane . . . . . . . . . . . 17 


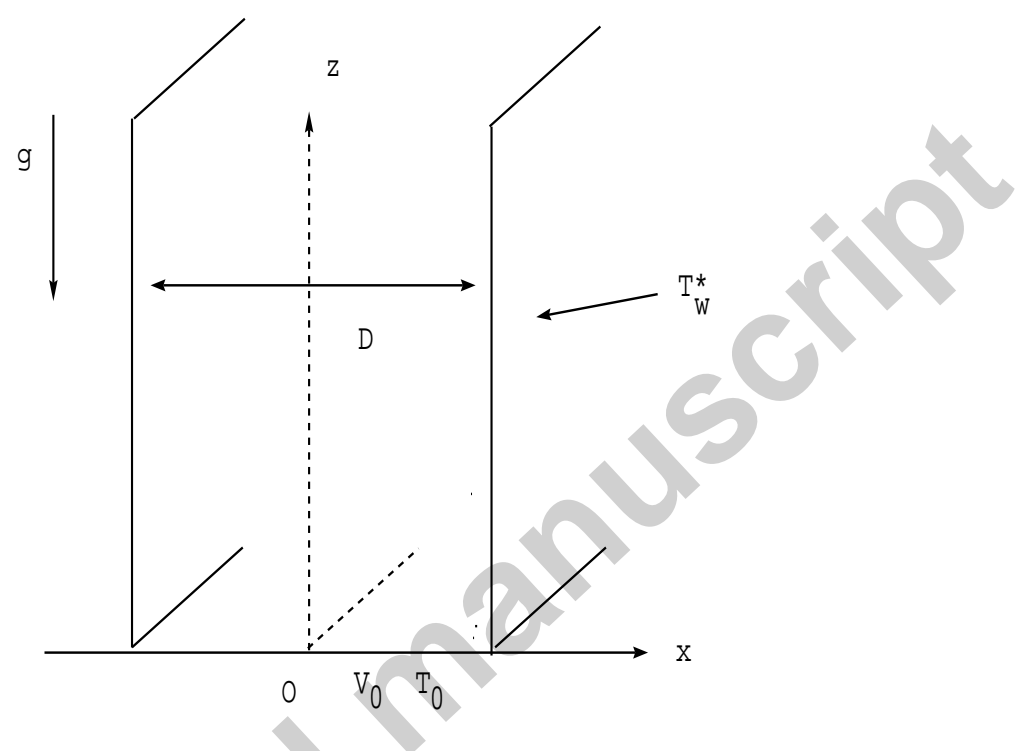

Figure 1: Schematic representation of system under study :Two parallel vertical plates 


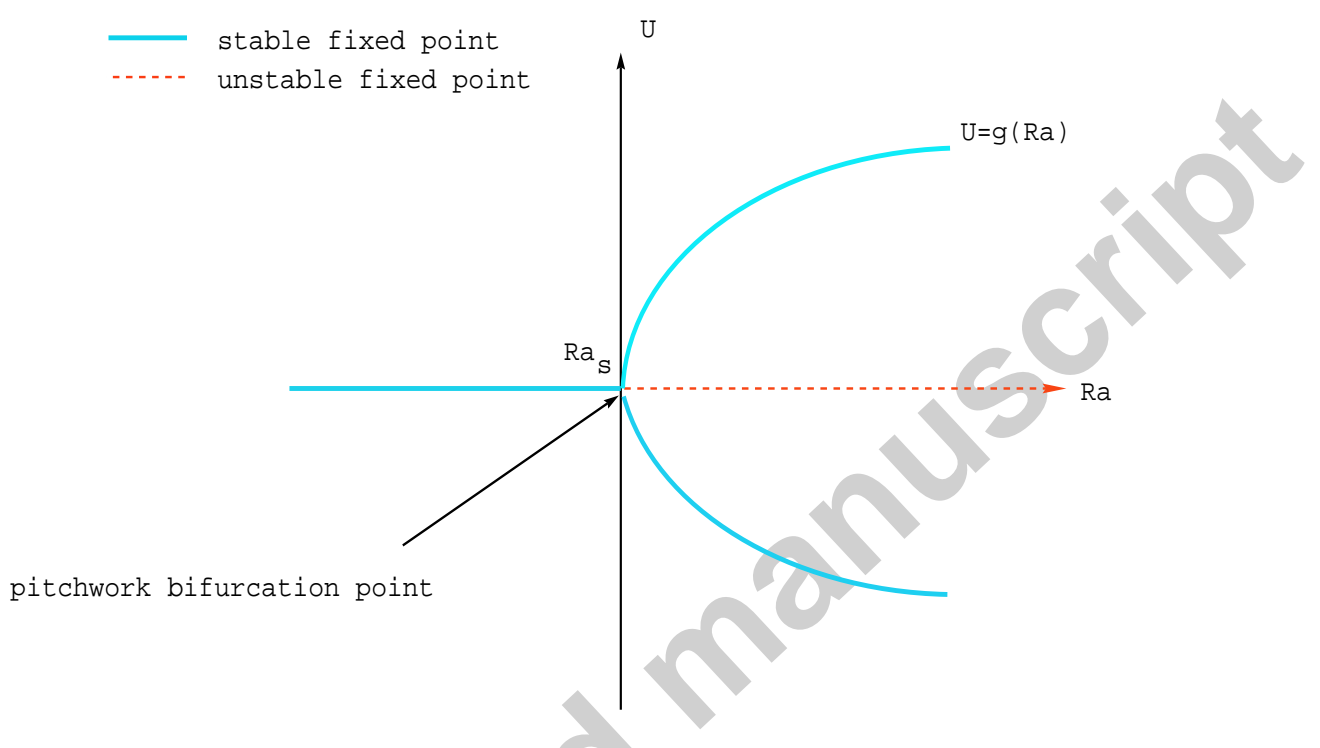

Figure 2: Bifurcation diagram on the center manifold in the $R a-U$ plane 


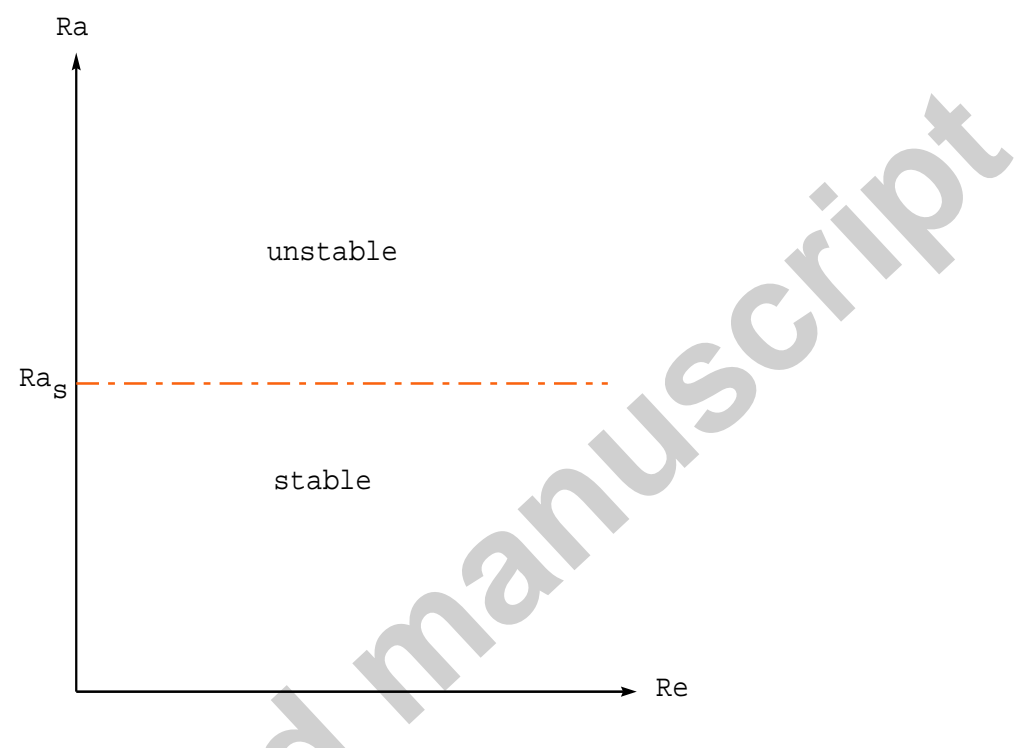

Figure 3: The instability boundary on the Re - Ra plane 